EPJ Web of Conferences 63,01020 (2013)

DOI: 10.1051 /epjconf/ 20136301020

(C) Owned by the authors, published by EDP Sciences, 2013

\title{
Giant Dipole Resonance in A 144 mass region
}

\author{
Ish Mukul ${ }^{1, a}$, P. Sugathan ${ }^{1}$, I. Mazumdar ${ }^{2}$, J. Gehlot ${ }^{1}$, G. Mohanto ${ }^{1}$, Maninder Kaur ${ }^{3}$, A. K. Rhine Kumar ${ }^{4}$, N. \\ Madhavan ${ }^{1}$, S. Nath ${ }^{1}$, R. Dubey ${ }^{1}$, D. A. Gothe ${ }^{2}$, P. Arumugam ${ }^{4}$, and A. Roy ${ }^{1}$ \\ ${ }^{1}$ Inter University Accelerator Centre, Aruna Asaf Ali Road, New Delhi, India \\ ${ }^{2}$ Department of Nuclear and Atomic Physics, Tata Institute of Fundamental Research, Homi Bhabha Road, Mumbai, India \\ ${ }^{3}$ Department of Physics, Panjab University, Chandigarh, India \\ ${ }^{4}$ Department of Physics, Indian Institute of Technology Roorkee, Roorkee, Uttarakhand, India
}

\begin{abstract}
Exclusive measurement of giant dipole resonance (GDR) $\gamma$ rays has been performed in ${ }^{144} \mathrm{Sm}$ nucleus which was populated at near barrier energy using the heavy ion reaction of ${ }^{28} \mathrm{Si}$ beam on ${ }^{116} \mathrm{Cd}$ target. GDR $\gamma$ rays were detected in coincidence with low energy $\gamma$ rays using 32 elements $4 \pi$ sum-spin spectrometer. The ${ }^{144} \mathrm{Sm}$ nucleus was populated at an excitation energy of $68 \mathrm{MeV}$ in the temperature range of 1.1-1.3 MeV. The measured GDR widths in this temperature range are consistent with the Kusnezov's parametrization.
\end{abstract}

\section{Introduction}

The phenomenon of Giant Dipole resonance (GDR) built on excited states $[1,2]$, offers unique possibility to study the fundamental properties of hot rotating nuclei. GDR characteristics, viz. centroid, width, strength etc. are found to be dependent on the temperature $(T)$ and angular momentum $(J)$. Using high efficiency detector array setup, a few experiments $[3,4]$ have been carried exclusively to study the dependence of temperature and angular momentum on GDR observables. Heavy ion fusion reactions impart a large amount of energy and angular momentum to the internal degrees of freedom in hot rotating nuclei. The quadrupole deformation experienced by the compound nuclear system can be inferred from the splitting of GDR strength function. Though several theoretical models have been proposed to explain the effect of angular momentum on GDR widths, a parametrization proposed by Kusnezov et al. [5] has been successfully used in fitting the evolution of GDR widths in 1-2 MeV temperature range in different mass regions. Bhattacharya et al. [6] have used this phenomenological function to scale experimental widths in recent experiments spanning different mass regions. In these measurements, it is necessary to have high resolution of angular momentum selection to decouple the effect of $J$ and $T$ mixing at a given excitation energy. The multiplicity filter with high efficiency and granularity plays a crucial role in such experiments. Increase in solid angle coverage leads to better selection of $J$ windows. Using the $4 \pi$ spin-spectrometer and high energy $\gamma$ spectrometer at Inter University Accelerator Centre (IUAC), we have studied the GDR characteristics for ${ }^{144} \mathrm{Sm}$ [7] at wide range of $T$ and $J$ for which the ground state systematics are already known [8]. In this work, we aim to extract GDR widths

\footnotetext{
ae-mail: ishmukul@gmail.com
}

from ${ }^{144} \mathrm{Sm}$ nucleus by populating it at near barrier energies. Thus, we could map the evolution of GDR widths as a function of angular momentum as well as temperature. In this paper, we present initial observations of the effect of angular momentum on GDR width in ${ }^{144} \mathrm{Sm}$ at near barrier energy and low temperatures.

\section{Experiment details}

The experiment was performed at the 15UD Pelletron accelerator facility at IUAC, New Delhi. The accelerated DC beam of ${ }^{28} \mathrm{Si}$ at $125 \mathrm{MeV}$ energy was bombarded on ${ }^{116} \mathrm{Cd}$ target to form the compound nucleus $(\mathrm{CN}){ }^{144} \mathrm{Sm}$. The target was prepared at target laboratory of IUAC by rolling to a thickness of $\sim 1.8 \mathrm{mg} / \mathrm{cm}^{2}$. The targets were self supporting with more than $98 \%$ enrichment. The ${ }^{144} \mathrm{Sm}$ nuclei were populated at an excitation energy of $68 \mathrm{MeV}$. The high energy $\gamma$ rays were detected in High Energy Gamma Ray Spectrometer (HiGRaSp) [9] which consists of a single crystal of $\mathrm{NaI}(\mathrm{Tl})$ in the centre surrounded by four plastic scintillator detectors. Plastic detectors were used as active shield for cosmic background reduction. The $\mathrm{NaI}(\mathrm{Tl})$ has a dimension of $25.4 \mathrm{~cm} \times 30.5 \mathrm{~cm}$ and is coupled with seven Photo Multiplier Tubes (PMT) operating at a voltage of $+800 \mathrm{~V}$. All the PMTs were gain matched and the dynode signals were fed to spectroscopic amplifiers. The summed output from these amplifiers was fed to Analog to Digital Converter (ADC) as energy signal. Anode signals from all the PMTs were amplified using Fast amplifiers and fed to Constant Fraction Discriminators (CFD) providing fast timing signals for starting various Time to Amplitude Converters (TAC).

HiGRaSp was positioned at a distance of $80 \mathrm{~cm}$ from the target centre at an angle of $90^{\circ}$ with respect to the beam 

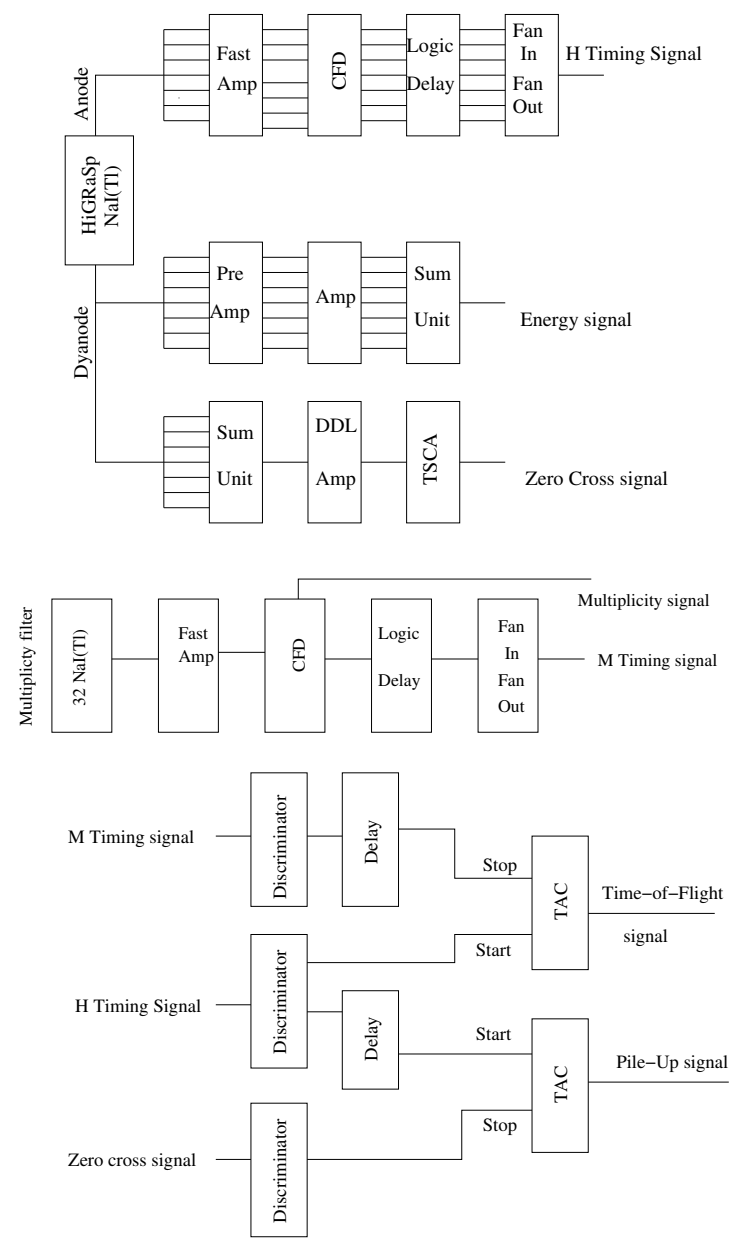

Figure 1. Block diagram of electronics circuit used in the experiment.

direction. Laboratory $\gamma$ ray sources were used for calibration and extraction of experimental response function. The response function of detector for mono-energetic $\gamma$ rays was generated using monte-carlo simulation package GEANT4 [10]. The dynamic range of HiGRaSp was set at $34 \mathrm{MeV}$. Energy gain was monitored in-beam using the neutron capture peak of $6.83 \mathrm{MeV}$.

Low energy $\gamma$ rays from the fusion reaction were detected using the 32 element $4 \pi$ sum-spin spectrometer [11]. In the present experiment, 27 detectors were used covering nearly $82 \%$ of $4 \pi$ solid angle. An energy threshold of $100 \mathrm{keV}$ was set for each detector. A Time-of-Flight (TOF), set between HiGRaSp and sum-spin spectrometer, separated neutron and $\gamma$ events. To filter piled up events due to high count rate, a Pile-Up (PU) circuit was used implementing the zero cross-over technique. The block diagram of electronics used for processing signals from HiGRaSp and multiplicity filter is shown in fig. 1. The online data was recorded and analysed using the software package CANDLE [12], developed at IUAC. The raw 2D spectra of Energy vs TOF and Energy vs PU are shown in figures 2 and 3 , respectively.

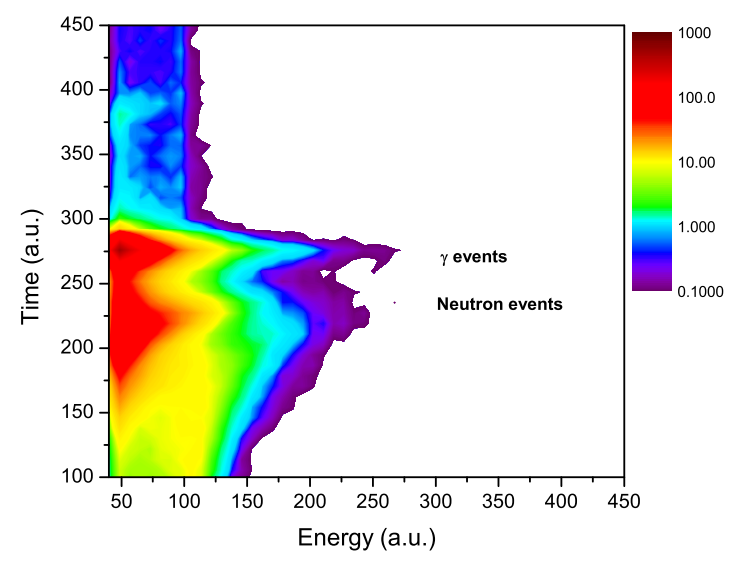

Figure 2. A time of flight (TOF) 2D spectrum showing prompt $\gamma$ events and other delayed events for $125 \mathrm{MeV}$ beam energy. The spectrum is gated with the condition of fold 6 and above.

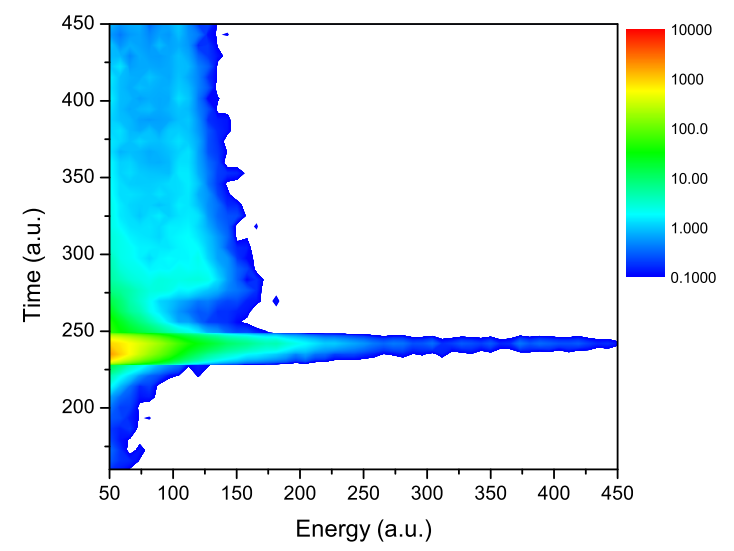

Figure 3. A Pile-Up 2D spectrum showing piled up events and good events. A tight gate was used on good events for cleaning the high energy $\gamma$ spectra.

\section{Data Reduction \& Analysis}

Various 1D histograms were generated offline using CANDLE. The fold distribution at beam energy of $125 \mathrm{MeV}$ is shown in fig. 4. Average angular momentum for different fold bins were calculated by extracting multiplicity distributions for those fold distributions. This was accomplished in the following way. The response function of multiplicity was generated using the iterative algorithm prescribed in Ref. [13]. According to this method, the probability of $M$ number of $\gamma$ rays hitting $F$ number of detectors out of total $N$ number of detectors is given by

$$
\begin{gathered}
S(F, M)=a S(F, M-1)+b S(F-1, M-1)+c S(F-2, M-1) \\
a=1-(N-F) \omega(1+\epsilon F / N-1) \\
b=(N-F+1) \omega(1-\epsilon(N-2 F+1) /(N-1)) \\
c=(N-F+2) \omega \epsilon((N-F+1) /(N-1))
\end{gathered}
$$

where $\omega$ is the cross talk probability and $\epsilon$ is the efficiency of each detector. Simulated fold distributions were generated using following equation. 


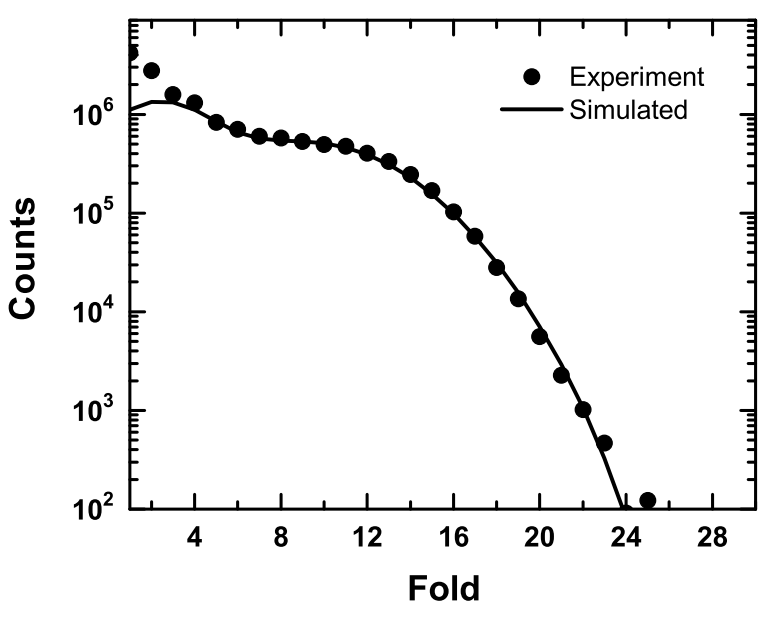

Figure 4. Experimental fold distribution at $125 \mathrm{MeV}$ beam energy data. Solid line is the best fit of simulated fold distribution.

$$
P(F)=\sum_{M=0}^{M_{\max }} S(F, M) P(M)
$$

The form of multiplicity distribution used in this method was assumed to be a modified fermi function and was given by

$$
P(M)=\frac{2 M+1}{1+\exp \left(\frac{M-M_{0}}{\delta M}\right)}
$$

where $M_{0}$ and $\delta M$ are free parameters. The simulated fold distribution was compared with the experimental fold distribution by varying the $M_{0}$ and $\delta M$ and minimizing the $\chi^{2}$. For $125 \mathrm{MeV}$ beam data, $M_{0}=17$ and $\delta M=3.0$ were obtained. The experimental and simulated fold distributions are shown in fig. 4. The multiplicity distributions corresponding to different fold bins were generated as $P(M)=$ $S(F, M) P(F)$. Finally, the average of these distributions can be related to average angular momentum as $\langle J\rangle=2\langle M\rangle$ $+C$, with $C=4$.

High energy $\gamma$ ray spectra were extracted offline by using $\gamma$ gate in TOF 2D spectra (fig. 2) and PU gates in PU-2D spectra (fig. 3). The resulting spectra were gated with different fold bins. These spectra were also cleaned by plastic detector signal in anti-coincidence for cosmic rays rejection. The final resulting spectra were Doppler corrected and were binned in energy bin of $1 \mathrm{MeV}$.

A modified version of statistical model code CASCADE [14] incorporating two component lorentzian function for GDR was used for the statistical model calculations. Asymptotic level density parameter was fixed at the value of $\tilde{\mathrm{a}}=\mathrm{A} / 8.5 \mathrm{MeV}^{-1}$. The code has an option of six parameter fitting for two component lorentzian distribution of GDR, viz. centroid energy $\left(E_{1}, E_{2}\right)$, strength $\left(S_{1}, S_{2}\right)$ and width $\left(\Gamma_{1}, \Gamma_{2}\right)$. The CASCADE output, after varying GDR parameters, was folded with detector response and compared with the experimental spectra. Fit parameters were obtained by minimizing the $\chi^{2}$ in the

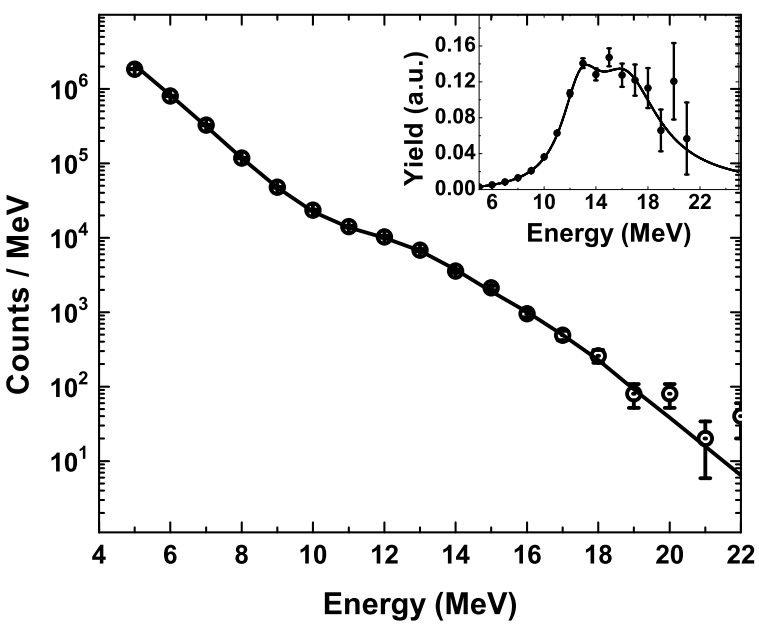

Figure 5. A cleaned high energy $\gamma$ ray spectra with a condition of fold 11-12 at beam energy of $125 \mathrm{MeV}$. Solid line is the CASCADE fit incorporating the two component GDR strength function. GDR strength in linear scale is shown in inset. Linearized plot is generated as $Y_{e x p} / Y_{t h} \times F_{2 L}$. See text for details.

energy range of 12-20 MeV. From these parameters, linearized GDR strength functions [15] were generated as $Y_{\text {exp }} / Y_{t h} \times F_{2 L}$, where $Y_{\text {exp }}$ is the experimental yield, $Y_{t h}$ is the CASCADE calculation folded with detector response and $F_{2 L}$ is the two component lorentzian function with each component given by

$$
F_{L}\left(E_{\gamma}, E_{C}, \Gamma\right)=\frac{\Gamma^{2} E_{\gamma}^{2}}{\left(E_{\gamma}^{2}-E_{C}^{2}\right)+\Gamma^{2} E_{\gamma}^{2}}
$$

A fold gated high energy $\gamma$ ray spectrum fitted with CASCADE output is shown in fig. 5. The GDR widths were extracted as FWHM of the GDR strength function as depicted in inset of fig. 5 .

\section{Results}

The experimental GDR widths $(\Gamma(T, J))$ at temperature $(T)$ and angular momentum $(J)$ have been extracted for ${ }^{144} \mathrm{Sm}$ and presented as a function of $J$ at the average temperature bins of 1.1 and $1.3 \mathrm{MeV}$. The experimental width has been compared with calculated GDR widths at given $T$ using the phenomenological function proposed by Kusnezov et al. which is based on thermal shape fluctuation model (TSFM). The GDR width at a finite $T$ and $J$ in liquid drop regime can be calculated as

$$
\Gamma(T, J)=\Gamma(T, 0)\left(1+\frac{1.8}{1+\exp \left(\frac{1.3-\xi}{0.2}\right)}\right)^{\frac{4 T_{0}}{T+3 T_{0}}}
$$

where

$$
\Gamma(T, 0)=\left(6.45-\frac{A}{100}\right) \ln \left(1+\frac{T}{T_{0}}\right)+\Gamma_{0}(A)
$$

Here $\Gamma_{0}(\mathrm{~A})$ is the width for the spherical shape of the nucleus and the value of $T_{0}$ used is $1 \mathrm{MeV}$. $\xi$ is the reduced angular momentum defined as $\xi=J / A^{5 / 6}$, which is 


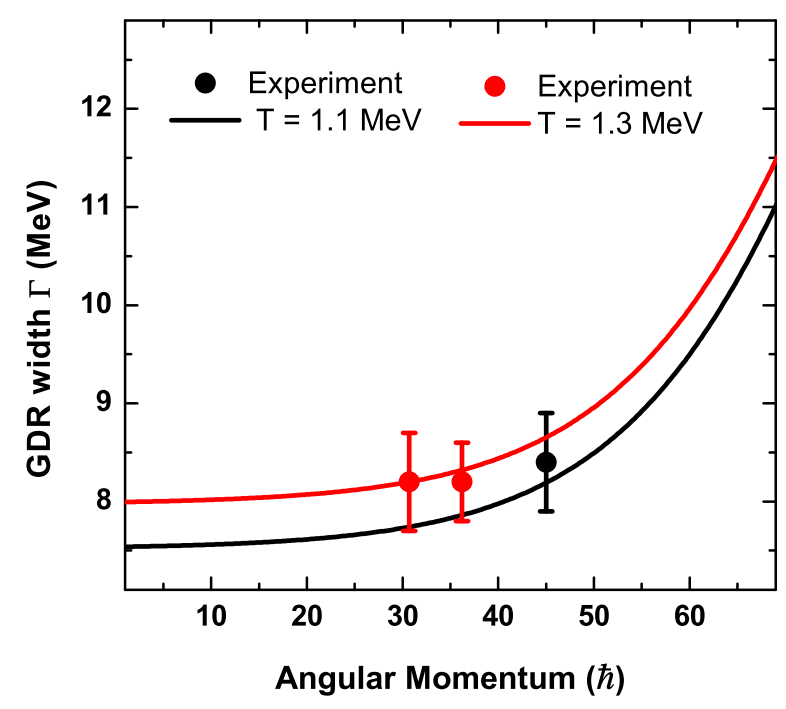

Figure 6. GDR width as a function of average angular momentum. Circles represent experimental data, and Solid lines are theoretical calculations for different $T$ values using the kusnezov's parametrization.

proportional to the rotational energy of the system. In these calculations, $\Gamma_{0}$ was adjusted to match the experimental width at low angular momentum and was fixed to be $3.8 \mathrm{MeV}$. The experimental GDR widths are consistent with the widths calculated using this parametrization, in the given temperature range as shown in fig. 6. The analysis of data at higher beam energies for the same reaction channel is under process.

\section{Summary}

Angular momentum gated GDR widths were studied in ${ }^{144} \mathrm{Sm}$ nucleus using heavy ion reaction of ${ }^{28} \mathrm{Si}+{ }^{116} \mathrm{Cd}$ at beam energy of $125 \mathrm{MeV}$. The compound nucleus was populated at near barrier energy to get the lowest temperatures. The experimentally extracted width is compared with theoretically calculated width using Kusnezov's parametrization. The theoretical predictions are con- sistent with experimental results for this nucleus. More experiments are planned to scan wider range of temperature and angular momentum for this nucleus.

\section{Acknowledgements}

We would like to thank the support of Pelletron group at IUAC for providing good quality beam throughout the experiments. One of the author (Ish Mukul) thanks UGC for providing fellowship under Senior Research Fellow scheme and CSIR for providing travel support for attending this conference.

\section{References}

[1] K.A. Snover, Ann. Rev. Nucl. Part. Sci. 36545 (1986)

[2] J.J. Gaardhoje, Ann. Rev. Nucl. Part. Sci. 42483 (1992)

[3] D. R. Chakrabarty et al., Nuc. Phys. A 770, 126 (2006).

[4] S. Bhattacharya et al., Phys. Rev. C 77024318 (2008)

[5] D. Kusnezov, Y. Alhassid, and K. A. Snover, Phys. Rev. Lett. 81, 542 (1998).

[6] S. Bhattacharya et al., Phys. Rev. C 78, 064601 (2008).

[7] Ish Mukul et al., Proceedings of the DAE Symp. on Nucl. Phys. 57, 284 (2012)

[8] P. Carlos et al., Nuc. Phys. A 225, 171 (1974).

[9] I. Mazumdar et. al., Nucl. Instrum. Methods Phys. Res. A 417, 297 (1998)

[10] S. Agostinelli et. al., Nucl. Instrum. Methods Phys. Res. A 506, 250 (2003)

[11] G. Anil Kumar et al., Nucl. Instr. Meth. A 611, 76 (2009)

[12] B. P. Ajith Kumar, E. T. Subramaniam, R. K. Bhowmik., Proceedings of the DAE Symp. on Nucl. Phys. (2001)

[13] A. Maj et al., Nuc. Phys. A 571, 185 (1994).

[14] F. Puhlhofer, Nuc. Phys. A 280, 267 (1977).

[15] Ish Mukul et al., Phys. Rev. C 88, 024312 (2013) 